\title{
RETINO-CHOROIDITIS RADIATA
}

\author{
BY \\ T. Hewitson Brown, M.B., Ch.B.(Edin.) \\ (FROM THE EYE CLINIC OF THE RIGSHOSPITAL IN \\ COPENHAGEN. CHIEF : PROFESSOR ROENNE)
}

THE following case of retino-choroidal atrophy presents such unusual features as to make it worth recording.

The patient, V.F., aged 47 years, government clerk, was admitted in June, 1937, to the Skin Department of this hospital, on account of alopecia areata of one year's duration. He stated that he had been short-sighted since his 13th year. In 1916 he joined the Danish Army, but shortly afterwards was admitted to hospital on account of symptoms suggestive of spondylitis tuberculosa. He was also found to be suffering from "choroiditis disseminata," for which he received 3 weeks' treatment and was discharged from the service. From that time until a year ago he remained in good health, and had noticed no alteration in his powers of vision. During the past year, apart from alopecia areata, he has suffered from frontal sinus infection and from sciatica. He states that several of his close relatives, including brothers and sisters, have died of tuberculosis.

Examination.-A well-built, intelligent man of healthy appearance. Extensive alopecia areata in the occipital region. Where hair is present it is partly white, partly pigmented, in patches.

Eyes.-Anterior segments normal. Tension normal. Fundi : the two eyes present an identical appearance. The discs are normal, with circumpapillary atrophy of one to one and a half discs' diameter; within the atrophic area there are small scattered spots of pigment. Arteries rather narrow. Partially confluent with the area of circumpapillary atrophy, extending peripherally almost to the equator, and centred on the retinal veins like beads on a string, there are numerous patches of choroidal atrophy, sometimes of dead white colour, sometimes showing narrowed remnants of the larger choroidal vessels; within these atrophic areas there are large prominent punctate masses of pigment which generally ensheathe the vessels but sometimes lie deep to them. The atrophic areas are one to one and a half discs' diameters in breadth and follow the distribution of the retinal veins so exactly that in many places where the latter bifurcate so do the former. They are in most instances bounded by retina of normal appearance, but here and there they are partially demarcated by a narrow zone of pigment. In addition, those parts of the veins where no atrophy is present are in many instances delineated by a sheath of pigment. One single atrophic patch is not centred accurately on 


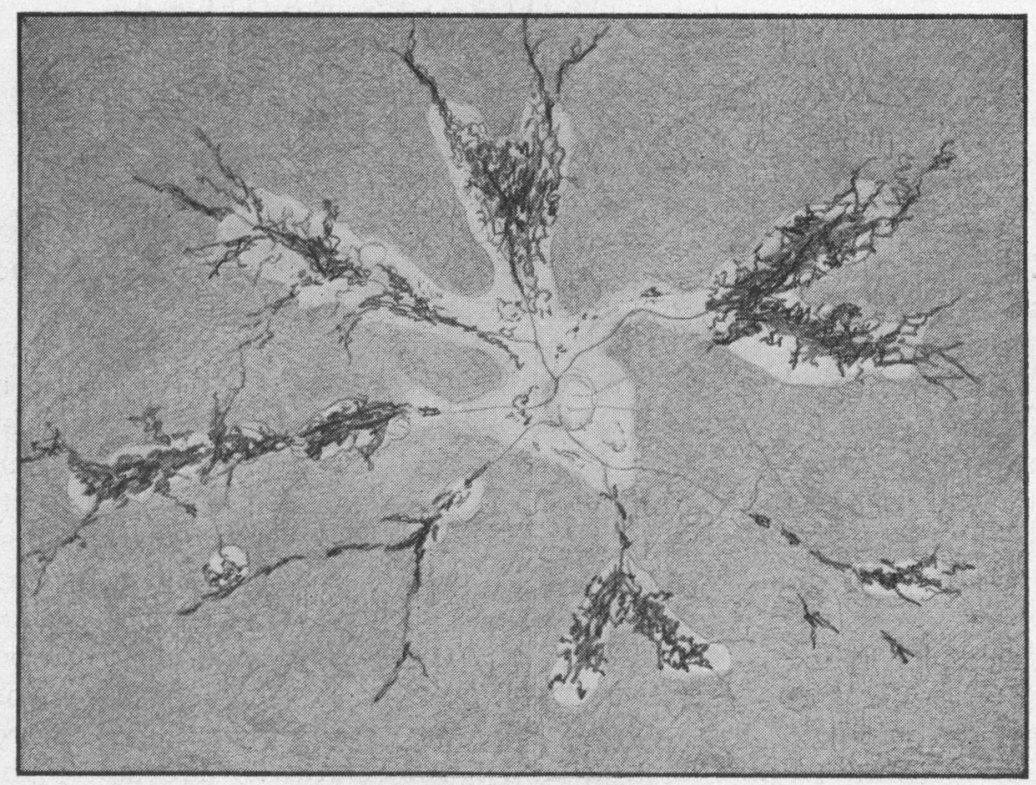

Right eye (indirect method).

a vein, but lies contiguous with it. Fields of vision : slightly contracted, with scotomata corresponding to the atrophic areas, but with no demonstrable nerve fibre bundle defects extending to the periphery.

$$
\begin{aligned}
\text { Vision : } 1912: & \text { r.e. } 6 / 60 ;-3.00 \text { D.sph. } 6 / 9 . \\
& \text { l.e. } 6 / 60 ;-3.00 \text { D.sph. } 6 / 9 . \\
1937: & \text { r.e. }-2.50 \text { D.sph. and }-1.00 \text { D.cyl. ax. } 25^{\circ} 6 / 9 . \\
& \text { l.e. }-2.50 \text { D.sph. and }-1.00 \text { D.cyl. ax. } 10^{\circ} 6 / 9 .
\end{aligned}
$$

von Pirquet test positive. No evidence of syphilis. Adaptation test not carried out on account of patient's refusal.

Commentary.-The unique feature of the case is the exact localisation of all the patches of choroidal atrophy along the course of the retinal veins. No similar case could be traced in the literature; only one case of active unilateral choroiditis (de Schweinit $z^{1}$ ) showed a similar distribution: " the exudate began 2 discs' diameter from the optic papilla and followed the sweep of the retinal vessels particularly as they passed downwards and outwards, as well as upwards."

The fields of vision, particularly the absence of nerve fibre bundle defects, prove that the innermost layer of the retina-that is, the layer in which the retinal veins lie-is relatively intact; and 
the presence of choroidal vessels in the atrophic patches proves that some at least of the choroid is also preserved. It is generally accepted that the original lesion in choroiditis lies in the chorio-capillaris, though occasionally similar lesions may occur in the retina (Gilbert ${ }^{2}$ ). Fuchs ${ }^{3}$, however, points out that we may easily derive a false impression as to the relative frequency of choroidal and retinal lesions from the fact that the latter are easily overlooked, whereas the former, since they cause gross pigmentary disturbances, are at once evident. The presence of such retinal lesions may be assumed (1) when the retina is particularly cloudy in the neighbourhood of the active focus, when there are small haemorrhages and when the retinal vessels are obscured, (2) in the presence of cloudiness of the retina at a distance from the focus, around the papilla and along the large vessels, (3) in the presence of sector-shaped defects in the fields of vision pointing to damage of the nerve fibres at the site of the focus, and (4) when permanent changes, confined to the pigment layer, remain after the active inflammation has subsided (Fuchs, 1.c.).

In the case under discussion we have unfortunately no record of the ophthalmoscopic appearance while the disease was active; after the passage of 21 years conditions (3) and (4) are not fulfilled.

Yet there can be no doubt that the retinal veins were responsible for the distribution of the retino-choroidal atrophy. Since there is no metabolic activity between the larger vessels and the surrounding tissues, there must have been some disease process occurring in the vessel walls themselves or in the accompanying lymphatics, which, spreading from them, affected chiefly the deeper layers of the retina and the superficial layers of the choroid. If this was the course of development of the atrophy, then the original lesion must have been located in the retina. The only other possibility is to assume that some wide-spread pathological process occurred in the choroid and retina, but that it entirely resolved except where the retinal veins exercised an unfavourable influence, which again could only be in the nature of disease of the vessel walls or of the lymphatics, creating a locus minoris resistentiae. In either case it is astonishing that so little damage should have been sustained by the nerve fibre layer of the retina.

The case is obviously and essentially different from " choroiditis disseminata" in which the haphazard distribution of the atrophic patches is a constant feature, though of course the pigment may commonly be found ensheathing the retinal veins.

Chorio-retinitis (Jensen) can hardly be compared with the disease under discussion; it is unilateral, generally confined to one juxta-papillary lesion, and invariably characterised by the permanence of the nerve fibre bundle defects. 
Several authors, particularly Japanese (Koyanagi ${ }^{4}$, Komoto $^{5}$ ), have noted a connection between uveitis, vitiligo and alopecia which is generally indistinguishable from alopecia syphilitica. This patient's skin condition was that of typical alopecia areata, also in the pigmentary disturbances which accompanied it; and the lapse of 20 years makes it extremely improbable that there is any factor common to his two diseases.

One disease, periphlebitis tuberculosa, is localised along the retinal veins in the manner mentioned; it is accompanied by vitreous haemorrhages and commonly leads to retinitis proliferans. This man, with his bad family history as regards tuberculosis, and with his positive reaction to tuberculin, may well have been the subject of a tuberculous periphlebitis which instead of taking its usual course and affecting the vitreous, has spread outwards towards the choroid; and this hypothesis is tentatively put forward as a possible explanation of the case.

I should like to express my thanks to Professor Roenne for his permission to publish this case. The accompanying sketch is also his work.

\section{Summary}

A case of retino-choroidal atrophy is described in which the atrophic areas are confined to the immediate proximity of the retinal veins. No similar case could be traced in the literature. One is compelled to conclude that it is a disease sui generis, for which the name retino-choroiditis radiata is suggested.

\section{REFERENCES}

1. de Schweinitz.-Ophthalmic Record, p. 23, 1909.

2. Gilbert's article in Schieck and Brückner's Kurzes Handbuch, Vol. 5, 1930.

3. Fuchs, E.-Zentralb. f. Augenheilk., Vol. VIII, p. 100, 1923.

4. Koyanagi.-Klin. Monatsbl. f. Augenheilk., Vol. LXXXII, p. 194, 1929.

5. $\mathrm{K}$ moto.-Klin. Monatsbl. f. Augenheilk., Vol. XLIX, pt. 1, 1911.

\section{TARSITIS SYPHILITICA}

BY

M. KhaLIL

CAIRO

\section{Introduction}

(a) Various syphilitic affections of the lid.

Quoting from Connell and others: It may be said that all cases of syphilis of the eyelid are unusual, because this disease rarely manifests itself by a lesion in this location. Zeissl reported that of 40,000 cases of syphilis examined, only eight had lesions of the 